\title{
INVENTORY CONTROL SYSTEM BASED ON CONTROL CHARTS TO IMPROVE SUPPLY CHAIN PERFORMANCES
}

\author{
Costantino, F. ; Di Gravio, G. ; Shaban, A. ${ }^{*, * *} \&$ Tronci, M. \\ "Department of Mechanical and Aerospace Engineering, University of Rome "La Sapienza", \\ Via Eudossiana 18, 00184 Rome, Italy \\ ** Industrial Engineering Department, Faculty of Engineering, Fayoum University, Fayoum, Egypt \\ E-Mail: francesco.costantino@uniroma1.it, giulio.digravio@uniroma1.it, \\ ahmed.shaban@uniroma1.it, massimo.tronci@uniroma1.it
}

\begin{abstract}
Inventory replenishment rules have been recognized as a major cause of the bullwhip effect and inventory instability in multi-echelon supply chains. There is a trade-off between bullwhip effect and inventory stability where mitigating the bullwhip effect through order smoothing might increase inventory instability. Therefore, there is a substantial need for inventory control policies that can cope with supply chains dynamics. This paper attempts to formulate an inventory control system based on a statistical control chart approach to handle the trade-off between order variability amplification and inventory stability. The proposed replenishment system, namely IR-SPC, incorporates individual control charts to control both the inventory position and the placed orders. A simulation study has been conducted to evaluate and compare the IR-SPC with a generalized order-up-to that has order smoothing mechanism. The comparisons showed that the IR-SPC outperforms both the smoothing order-up-to policy and the Min-Max inventory policy in terms of bullwhip effect and inventory performances.

(Received in June 2013, accepted in January 2014. This paper was with the authors 1 month for 2 revisions.)
\end{abstract}

Key Words: Multi-Echelon Supply Chain, Inventory Control System, Bullwhip Effect, Inventory Variance, Control Chart, Simulation

\section{INTRODUCTION}

In supply chains, the variability in the ordering patterns often increases as demand information travels upstream in the supply chain, from the retailer towards the factory and the suppliers (see Fig. 1). This phenomenon has been recognized as the bullwhip effect [1] and it has been observed in many industries [1-4]. Forrester [5] was mostly the first to study this problem using system dynamics and called it demand variability amplification. Other researchers developed simulation games to illustrate the bullwhip effect and its impacts in supply chains [6]. Towill et al. [7] indicated that the bullwhip effect could lead to stock-outs, capacity utilization problems, lower quality products, and considerable production/transport costs.

Lee et al. [1] identified five fundamental causes of the bullwhip effect: demand signal processing, non-zero lead-time, order batching, price fluctuations and rationing and shortage gaming. Of our particular interest is the demand signal processing in which demand is forecasted using a forecasting method, and then a replenishment rule (inventory control policy) is applied to identify when and how much to order. Burbidge's Law of Industrial Dynamics states that 'If demand is transmitted along a series of inventories using stock control ordering, then the amplitude of demand variation will increase with each transfer' [8]. Generally, the inventory control is a complex problem to address especially in multi-echelon supply chains where inventory managers must consider two important factors when making inventory replenishments [9]. First, a replenishment rule has an impact on order variability amplification (as measured by the bullwhip effect, i.e., the ratio of the variance of orders over 
the variance of demand). Second, the replenishment rule has an impact on the inventory stability (as measured by the inventory variance ratio, i.e., the ratio of net inventory variance over the variance of demand). The bullwhip effect mainly contributes to upstream costs, while the variance of net inventory determines the stage's ability to meet a desired service level in a cost-effective manner. This trade-off needs robust replenishment systems to balance the inventory and production costs whilst ensuring a customer service level [9]. Exhaustive research has been conducted to study the impact of various ordering policies on bullwhip effect, and to develop replenishment rules that can avoid the bullwhip effect [10-17]. In particular, extensive research has been focusing on developing smoothing ordering polices that are modified from the order-up-to policy with adding proportional controllers to restrict overreaction/underreaction to demand changes [17-20]. However, others have shown that decreasing the bullwhip effect largely might lead to increase the inventory variance and by collaborating in planning and replenishment both bullwhip effect mitigation and inventory stability can be achieved $[9,14]$.

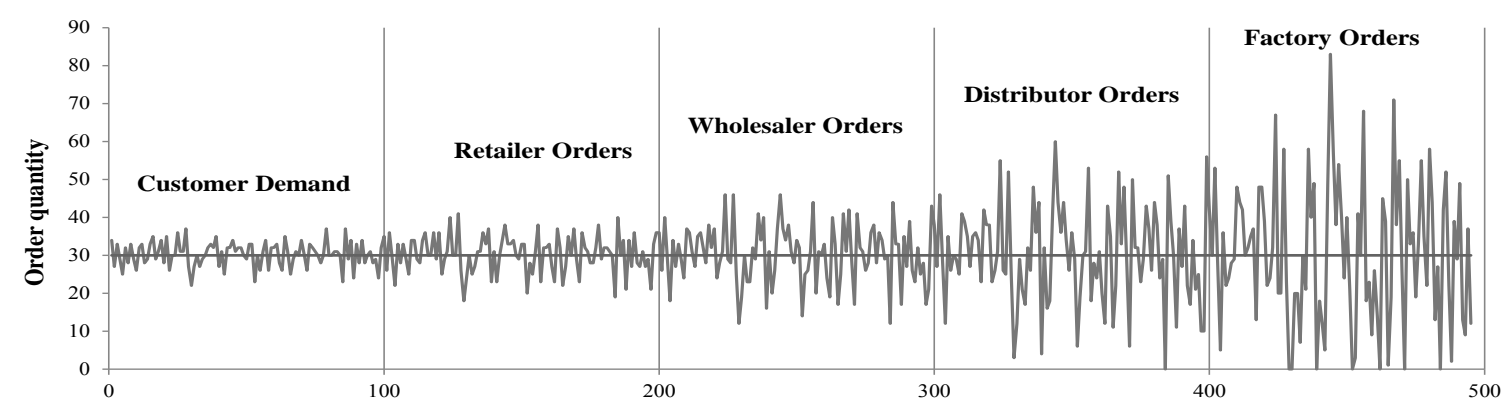

Figure 1: An example of demand amplification (bullwhip effect).

There is a substantial need for inventory control policies that can handle the trade-off between bullwhip effect and inventory performance without major implementation effort [12]. In practice, inventory control systems usually operate in dynamic environments like multiechelon supply chains in which calculating the optimal ordering quantities, deciding the proper reordering point, choosing the right inventory reviewing policies, and managing the safety stock are simply not enough in the these environments. In contrast, the inventory control policies should be smart enough to monitor the internal and external variables and propose the appropriate changes when it is needed. This is the target of this research where we attempt to develop a replenishment rule that can cope with supply chain dynamics.

Recently, the Statistical Process Control (SPC) which is used to detect unstable changes in processes has been employed to develop inventory replenishment policies for swiftly changing environments. Watts et al. [21] was mostly the first to employ a control chart approach for monitoring the performance of a reorder-point inventory system through monitoring stock-outs, demand and inventory turnover to identify the causes of system malfunctions. Pfohl et al. [22] developed a real-time inventory decision support system by employing the control charts for inventory level and demand along with a series of decision rules to help the inventory manager to determine the time and quantity to order. Cheng and Chou [23] extended the work of Pfohl et al. [22] by introducing the ARMA control chart for the market demand and the individual control chart with western electric rules for the inventory level. Lee and $\mathrm{Wu}[24]$ compared between traditional replenishment policies and SPC based replenishment policy and concluded that SPC based policy had shown better reduction in inventory variability than the traditional methods. With exception to Lee and $\mathrm{Wu}$ [24], the majority of the prior SPC work has considered a single echelon supply chain in order to evaluate the effectiveness of SPC ordering policies. Also, the previous work has neglected the effect of lead-time where they have assumed the immediate replenishments in their 
models. Furthermore, the performance measures that have been used in the previous work did not consider supply chain dynamics (i.e., bullwhip effect and inventory variance) under SPC policies. Most recently, Costantino et al. [12] have considered the above research gaps and proposed a SPC-based inventory control policy to improve supply chain dynamics. This research extends and compares their SPC model with the generalized order-up-to policy.

This research aims to develop a novel robust inventory control policy based on control charts to be used in dynamic and complex environments. A simulation methodology is adopted to conduct the study and to evaluate the performance of the proposed policy in a multi-echelon supply chain. Furthermore, a comparison will be conducted between the proposed policy and a generalized order-up-to policy in terms mainly of bullwhip effect, inventory performance and service level. The simulation results showed that the proposed policy performs better than the generalized order-up-to policy.

The paper is organized as follows. The next section describes the formulation of the proposed inventory control system. Section 3 describes the supply chain model, the generalized order-up-to policy and the performance measures. Sections 4 presents the simulation results and analysis. The discussion and conclusions are provided in sections 5 and 6 , respectively.

\section{PROPOSED REPLENISHMENT SYSTEM}

The replenishment system, depicted in Fig. 2, employs two individual control charts for monitoring demand changes and controlling inventory position, respectively. The first control chart is devoted to monitor the customer demand to make the appropriate changes whenever a considerable change has been detected. In addition, the demand control chart is complemented with a number of decision rules to decide about the out-of-control situation and how much to order under different situations. This is working as a forecasting system for the future demand. This action is the first stream in the flowchart in Fig. 2 that determines the base order quantity. The base order quantity will then be added to the required adjustment for the inventory position in order to constitute the final order to place.

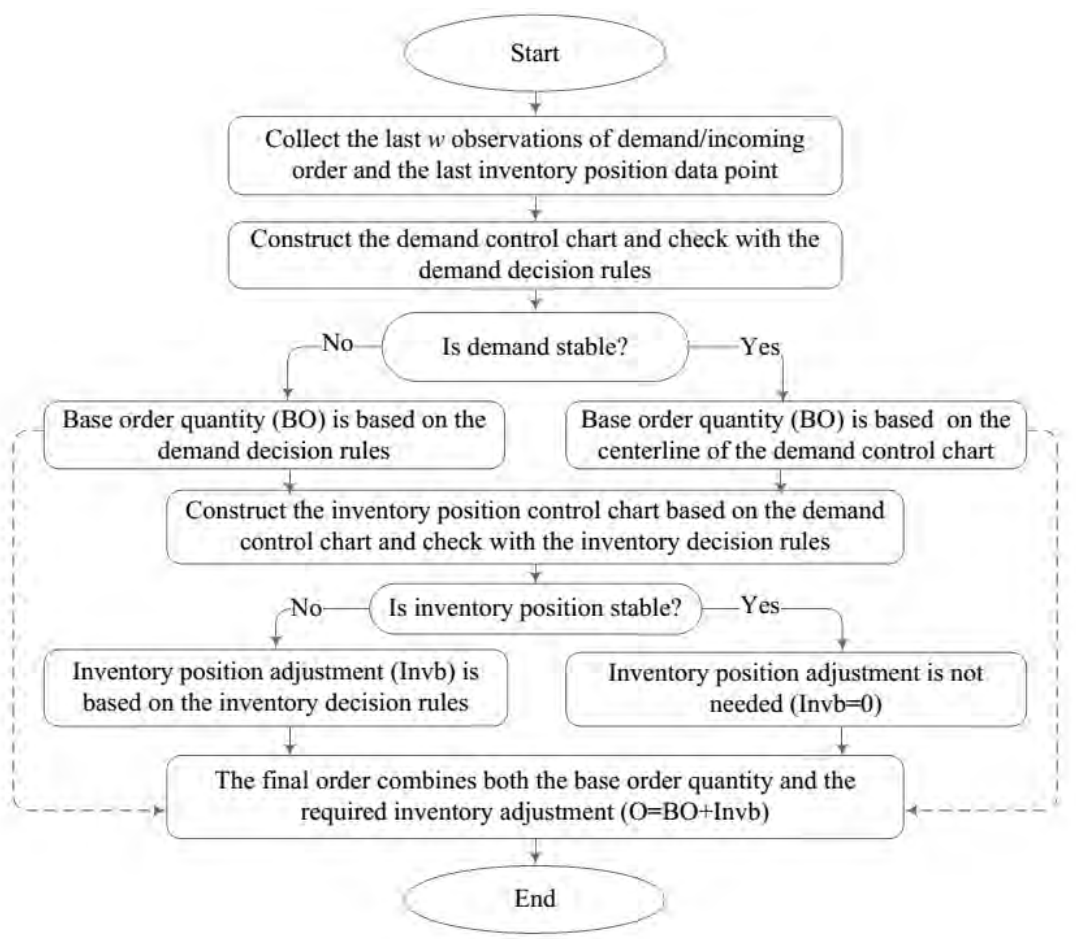

Figure 2: IR-SPC replenishment system flowchart. 
The second control chart is employed for monitoring and adjusting the inventory position. This control chart is used to identify whether the inventory position variable is in-control or not. In addition, this control chart is complemented with a number of decision rules to adjust the inventory position whenever it is needed. For example, if the current data point of inventory position is very low, then a decision rule will be needed to detect this situation and propose an amount of adjustment (inventory balance) to be added to the base order quantity.

\subsection{Control charts for the replenishment system}

A typical control chart is built based on the normality assumption and it generally consists of three basic elements: centreline that represents the average of the process variable, and lower and upper control limits [25]. If a process variable (e.g., customer demand process) is incontrol, then $99.73 \%$ of the demand data will be within the lower and upper control limits. The type of control chart that will be used for both demand and inventory position is called the individual control chart in which the sample size is equal to one [25]. The control limits of the demand and inventory position control charts can be calculated as follows in Table I.

Table I: Control charts calculations.

\begin{tabular}{|l|c|c|}
\hline \multicolumn{1}{|c|}{ Element } & Demand control chart & Inventory position control chart \\
\hline Upper control limit & $U C L_{D}^{i}=C L_{D}^{i}+3 \hat{\sigma}_{D}^{i}$ & $U C L_{I P}^{i}=C L_{I P}^{i}+3 \hat{\sigma}_{I P}^{i}$ \\
\hline Centreline & $C L_{D}^{i}=\frac{1}{w_{i}} \sum_{t-w_{i}+1}^{t} D_{i}^{t}$ & $C L_{I P}^{i}=L_{d}^{i} B O_{t}^{i}+S S_{i}^{t}$ \\
\hline Lower control limit & $L C L_{D}^{i}=C L_{D}^{i}-3 \hat{\sigma}_{D}^{i}$ & $U C L_{I P}^{i}=C L_{I P}^{i}-3 \hat{\sigma}_{I P}^{i}$ \\
\hline
\end{tabular}

The term $C L_{D}^{i}$ represents the centreline of the demand/incoming order control chart and is calculated based on the average of the last consecutive $w_{i}$ data points. The $L C L_{D}^{i}$ represents the lower control limit and is equal to the difference between $C L_{D}^{i}$ and $3 \hat{\sigma}_{D}^{i}$ where $\hat{\sigma}_{D}^{i}$ stands for the demand standard deviation over the time length $w_{i}$. The upper control limit $U C L_{D}^{i}$ equals the sum of $C L_{D}^{i}$ and $3 \hat{\sigma}_{D}^{i}$.

The centreline of the inventory position control chart is calculated based on the expected demand $\left(B O_{t}^{i}\right)$ multiplied by $L_{d}^{i}$, and the product of this multiplication is added to a safety stock component $\left(S S_{i}^{t}\right)$. For simplicity, we extend $L_{d}^{i}$ with $k_{i}$ to account for the safety stock and thus the centreline is $C L_{I P}^{i}=\left(L_{d}^{i}+k_{i}\right) B O_{t}^{i}$. This is similar to the calculation of the orderup-to level in the simple order-up-to policy as will be shown later. In this model and throughout this study, we set $\hat{\sigma}_{I P}^{i}=\eta_{i} \hat{\sigma}_{D}^{i}$ and for simplicity we set $\eta_{i}=1, \forall i$, thus, $\hat{\sigma}_{I P}^{i}=\hat{\sigma}_{D}^{i}$.

\subsection{Demand decision rules}

The demand decision rules are dependent on the last observations on the control chart. This is working as a forecasting method. If the demand is in-control and no change in its level, then the base order quantity should be based on the centreline of the demand control chart. Otherwise, if the demand is out-of-control, then the order quantity should be altered as follows.

Rule 1: If $q_{i}$ points of the last consecutive $Q_{i}$ data points of incoming order are above a defined safety zone between $C L_{D}^{i}-K_{D}^{i} \hat{\sigma}_{D}^{i}$ and $C L_{D}^{i}+K_{D}^{i} \hat{\sigma}_{D}^{i}$, then the base order quantity $\left(B O_{t}^{i}\right)$ is calculated based on the average of the last $n_{i}$ data points as in eq. (1). 


$$
B O_{t}^{i}=\operatorname{Max}\left\{C L_{D}^{i}, \frac{1}{n_{i}} \sum_{t-n_{i}+1}^{t} I O_{t}^{i}\right\}
$$

Rule 2: If $q_{i}$ points of the last consecutive $Q_{i}$ data points of incoming order are below the defined safety zone, then the base order quantity $\left(B O_{t}^{i}\right)$ is calculated based on eq. (2).

$$
B O_{t}^{i}=\operatorname{Min}\left\{C L_{D}^{i}, \frac{1}{n_{i}} \sum_{t-n_{i}+1}^{t} I O_{t}^{i}\right\}
$$

Rule 3: If the above conditions (Rules $1 \& 2$ ) are not satisfied, then the base order quantity is set equal to the centreline of the demand control chart as shown in eq. (3).

$$
B O_{t}^{i}=C L_{D}^{i}
$$

\subsection{Inventory position decision rules}

The inventory position decision rules depend on the last observation of inventory position $\left(I P_{t}^{i}\right)$ which contains information about the net inventory $\left(N I_{t}^{i}\right)$ and supply line $\left(S L_{t}^{i}\right)$. We define a target range for the inventory position and thus if $I P_{t}^{i}$ is within this range, then there is no need for inventory adjustment, otherwise, inventory adjustment should be added to $B O_{t}^{i}$.

Rule 1: If $I P_{t}^{i}$ is above the upper limit of a safety zone $\left(C L_{I P}^{i}+K_{I P}^{i} \sigma_{I P}^{i}\right)$, then a negative inventory balance ( $\operatorname{Inv} b_{t}^{i}$ ) should be added to the base order quantity as shown in eq. (4).

$$
\operatorname{Inv} b_{t}^{i}=C L_{I P}^{i}+K_{I P}^{i} \sigma_{I P}^{i}-I P_{t}^{i}
$$

Rule 2: If the last observation, $I P_{t}^{i}$, is below the safety zone $\left(C L_{I P}^{i}-K_{I P}^{i} \sigma_{I P}^{i}\right)$, then a positive inventory balance should be added to the base order quantity (eq. (5)).

$$
\operatorname{Inv} b_{t}^{i}=C L_{I P}^{i}-K_{I P}^{i} \sigma_{I P}^{i}-I P_{t}^{i}
$$

Rule 3: If the last observation, $I P_{t}^{i}$, is within the safety zone (i.e., $\left.C L_{I P}^{i}-K_{I P}^{i} \sigma_{I P}^{i} \leq I P_{t}^{i} \leq C L_{I P}^{i}+K_{I P}^{i} \sigma_{I P}^{i}\right)$, then there is no need for inventory balance: $I n v b_{t}^{i}=0$.

The final order $\left(O_{t}^{i} \geq 0\right)$ to be placed by echelon $i$ at the end of time $t$ should be equal to the sum of the base order quantity and the inventory balance as shown in eq. (6).

$$
O_{t}^{i}=\operatorname{Max}\left\{B O_{t}^{i}+\operatorname{Inv} b_{t}^{i}, 0\right\}
$$

\section{SUPPLY CHAIN MODELING AND SIMULATION}

We model a single product multi-echelon supply chain that consists of a customer, a retailer, a wholesaler, a distributor, and a factory to evaluate the IR-SPC policy (see Fig. 3). This model has been adopted widely in many similar investigations $[15,16]$. It is assumed that all echelons have unlimited stocking capacity, both the supplier and the factory have unlimited capacity, and the lead-times are deterministic and fixed across the supply chain with ordering lead-time of one and delivery lead-time $\left(L_{d}^{i}\right)=2$. Each echelon $i$ can apply either the generalized OUT or the IR-SPC policy but all echelons in the supply chain will apply the same policy. 


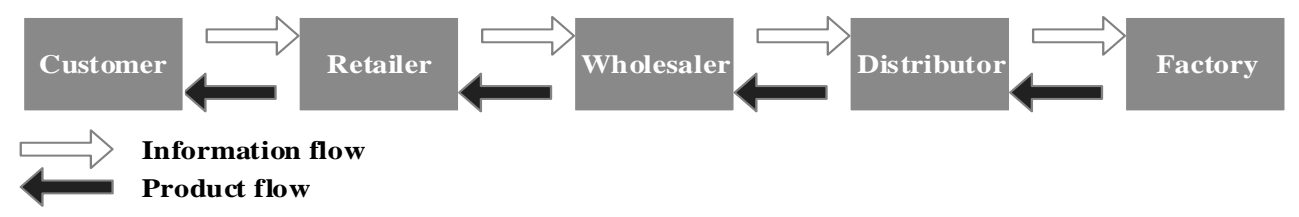

Figure 3: A multi-echelon supply chain.

\subsection{Generalized order-up-to policy}

The periodic review order-up-to (OUT) has been common in the bullwhip literature because of its optimality in terms of inventory variance and its popularity in practice [20]. A variation of this policy is used here to generate the historical data required to initiate the IR-SPC and to validate the effectiveness of the IR-SPC. In the simple order-up-to, at the end of each review period $(R)$, an order $O_{t}^{i}$ is placed whenever the inventory position $I P_{t}^{i}$ is lower than a target level $S_{t}^{i}$. The review period is considered to be equal to one (i.e., $R=1$ ) for this model. The simple order-up-to policy can be represented mathematically as follows in eqs. (7-10).

$$
\begin{gathered}
O_{t}^{i}=\operatorname{Max}\left\{S_{t}^{i}-I P_{t}^{i}, 0\right\} \\
I P_{t}^{i}=S_{t-1}^{i}-I O_{t}^{i} \\
S_{t}^{i}=L \hat{D}_{t}^{i}+S S_{t}^{i} \\
\hat{D}_{t}^{i}=\frac{1}{m_{i}} \sum_{j=1}^{m_{i}} I O_{t-j+1}^{i}
\end{gathered}
$$

The target inventory position $S_{t}^{i}$ is calculated based on the demand forecast over the leadtime. The moving average method (with the parameter $m_{i}$ ) is employed to calculate the expected demand because of its popularity in research and in practice as well $[13,20]$. In this model, we have considered the safety stock by extending the lead-time by $k_{i}[15,18]$ so that $S_{t}^{i}$ can be reformulated as shown below in eq. (11). This is similar to what we have done with the centreline of the inventory position control chart.

$$
S_{t}^{i}=\left(L+k_{i}\right) \hat{D}_{t}^{i}
$$

It can be seen that the simple order-up-to has a rigid structure to allow order smoothing and therefore some authors have proposed to incorporate proportional controller in order to control the inventory position variation as shown in eqs. (12-13). In other words, an order quantity by the order-up-to can be decomposed into three parts: the demand forecast, the gap between target and actual inventory levels, and the gap between target and actual supply lines as shown in eq. (12). This replenishment rule can be modified by incorporating two proportional controllers for inventory and supply line errors as shown in eq. (13).

$$
\begin{gathered}
O_{t}^{i}=\left(L_{d}^{i}+R+k_{i}\right) \hat{D}_{t}^{i}-N I_{t}^{i}-S L_{t}^{i} \\
O_{t}^{i}=\hat{D}_{t}^{i}+L_{d}^{i} \hat{D}_{t}^{i}-N I_{t}^{i}+k_{i} \hat{D}_{t}^{i}-S L_{t}^{i} \\
O_{t}^{i}=\hat{D}_{t}^{i}+\frac{L_{d}^{i} \hat{D}_{t}^{i}-N I_{t}^{i}}{T n}+\frac{k_{i} \hat{D}_{t}^{i}-S L_{t}^{i}}{T S}
\end{gathered}
$$

The term $L_{d}^{i} \hat{D}_{t}^{i}$ denotes the target supply line $\left(T S L_{t}^{i}\right), k_{i} \hat{D}_{t}^{i}$ denotes the target net inventory $\left(T N I_{t}^{i}\right)$, and both $T n$ and $T s$ are the proportional controllers. This ordering rule has been proposed as a mitigation solution for bullwhip effect by controlling both $T n$ and $T s[17,18]$. 
In this model, for simplicity, we consider $T n=T s$. Accordingly, the replenishment rule can be written in a general form in eq. (14) in which setting $T n=T s=1$ turns the ordering rule into a standard order-up-to policy. The standard order-up-to is a special case of the Min-Max inventory policy in which both the maximum and minimum inventory levels are equal.

$$
O_{t}^{i}=\operatorname{Max}\left\{\hat{D}_{t}^{i}+\frac{T N I_{t}^{i}-N I_{t}^{i}}{T n}+\frac{T S L_{t}^{i}-S L_{t}^{i}}{T n}, 0\right\}
$$

\subsection{Performance measures}

The performance of the supply chain under the different ordering policies will be evaluated through quantifying the ordering and inventory performances across the supply chain. The ordering behaviour can be evaluated by estimating: average order level and total variance amplification $\left(T V A_{i}\right)$. The $T V A_{i}$ (bullwhip effect) is used to quantify the demand variance amplification across the supply chain [26]. The $T V A_{i}$, as shown in eq. (15), can be estimated in terms of the ratio of orders variance divided by the corresponding orders average at echelon $i$ to the customer demand variance divided by the average demand $[13,18,20]$.

$$
T V A_{i}=\frac{\sigma_{O_{i}}^{2} / \mu_{O_{i}}}{\sigma_{D}^{2} / \mu_{D}}
$$

The inventory performance can be evaluated by estimating: average net inventory, inventory variance ratio, and average service level. The inventory variance ratio, as shown in eq. (16), represents the ratio of the actual net inventor variance $\left(\sigma_{N I_{i}}^{2}\right)$ to the customer demand variance [27].

$$
\operatorname{Inv} R_{i}=\sigma_{N_{i}}^{2} / \sigma_{D}^{2}
$$

The average service level ( $A S L_{i}$ ), as shown in eq. (18), quantifies the percentage of items delivered immediately by echelon $i$ for the order of echelon $i-1$ [28]. Service level $\left(S l_{t}^{i}\right)$ is computed over the effective delivery time (i.e., $I O_{t}^{i}>0$ ) as shown in eq. (17), where $S R_{t}^{i}$ stands for the shipment from echelon $i$ to echelon $i-1$ at $t, B_{t-1}^{i}$ stands for the initial backlog at echelon $i$ at $t$, and $I O_{t}^{i}$ is the incoming order to echelon $i$ at time $t$.

$$
\begin{gathered}
S l_{t}^{i}=\left\{\begin{array}{lll}
\frac{S R_{t}^{i}-B_{t-1}^{i}}{I O_{t}^{i}} \times 100 & \text { if } & S R_{t}^{i}-B_{t-1}^{i}>0 \\
0 & \text { if } \quad S R_{t}^{i}-B_{t-1}^{i} \leq 0
\end{array}\right. \\
A S L_{i}=\frac{\sum_{t=1}^{T_{e f f}} S l_{t}^{i}}{T_{\text {eff }}}
\end{gathered}
$$

\section{SIMULATION RESULTS AND ANALYSIS}

A simulation model has been developed for the above supply chain model using SIMUL8. For all simulation experiments, the simulation model is run for 20 replications of 1400 periods each. Each simulation run consists of four stages, the first stage is a warm-up period for the generalized order-up-to policy, and the second stage is the effective simulation run that the performance of the order-up-to will be collected over it. Then, another warm-period for the IR-SPC policy is considered, followed by an effective simulation run for the IR-SPC. 
Both warm-up periods are set to be of the same length (i.e., 200 periods), and both effective simulation runs are also set to be of the same length (i.e., 500 periods). The customer demand pattern is assumed to follow the normal distribution with a mean of 100 and a standard deviation of 10 .

\subsection{Comparing IR-SPC with smoothing order-up-to policy}

In this section, we compare IR-SPC with smoothing OUT in a multi-echelon supply chain. The parameters of the smoothing OUT are set to achieve the highest performance, in terms of bullwhip effect and inventory variance, by selecting a large value for the moving average parameter [13] as well as using recommended values for both the controller parameters [9] as follows: $T n=1.618, m_{i}=30$, and $k_{i}=1$. The IR-SPC parameters are set as follows: $w_{i}=30$, $K_{I P}^{i}=2.0, K_{D}^{i}=2.0, q_{i}=3, Q_{i}=4$ and $n_{i}=w_{i} / 2$. The value of $m_{i}$ is selected to be equal to $w_{i}$ in order to make a consistent comparison with the IR-SPC.

The results of a simulation run is presented in Fig. 4 in which the ordering and inventory performance of the factory under each ordering policy over the last 200 periods of the effective simulation run are plotted. We selected the factory as it is the highest level in the supply chain and thus the most exposed to the bullwhip effect [26]. The factory orders are compared to the customer demand whereas the factory inventory is compared to the distributor order. The IR-SPC policy shows a stable order rate over the simulation time with infrequent changes in comparison to smoothing OUT. Furthermore, the inventory level seems to be more smoother under IR-SPC.

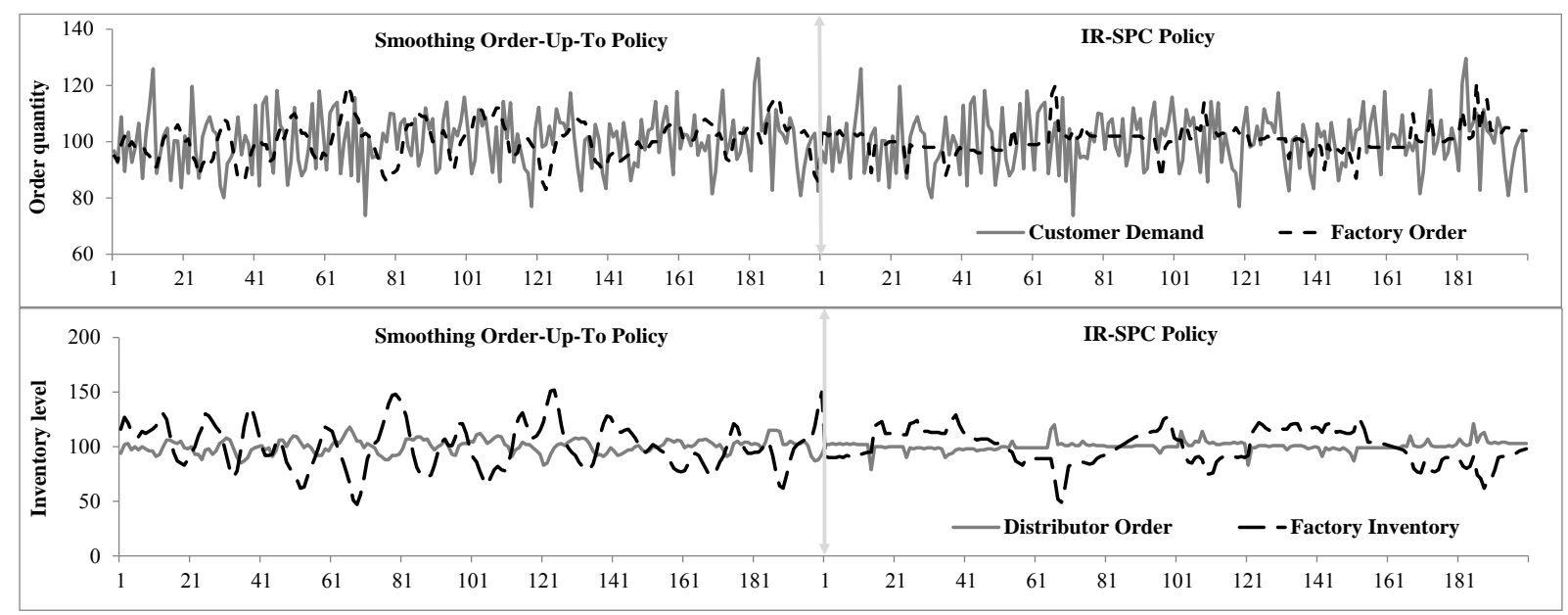

Figure 4: The performance of the factory under both smoothing OUT and IR-SPC.

The $T V A_{i}$ measure with $95 \%$ confidence level, before and after applying the IR-SPC policy, is summarized in Table II. The results reveal that both the replenishment policies are successful to eliminate the bullwhip effect (i.e., $T V A_{i}<1, \forall i$ ). It can also be observed that the bullwhip effect under both replenishment policies is initially decreasing as orders moves from the retailer to the wholesaler and then increasing again in the upstream direction. However, it is clear that the IR-SPC realizes a lower $T V A_{i}$ throughout the supply chain compared to OUT. The major reduction in the bullwhip effect under the IR-SPC is achieved at the retailer which places orders with a variability less than 0.331 of the demand variability. Both of the policies stabilize at the same average ordering level (100 units). Although we selected heuristic values for the IR-SPC, it can be concluded that the IR-SPC outperforms the optimized order-up-to and thus applying IR-SPC allows the upstream echelons to save capacity costs. 
The results in Table II show that both replenishment policies are successful to stabilize at the same average inventory level $(\sim 100)$, and the same average service level $\left(A S L_{i}=100 \%\right)$, throughout the supply chain. The IR-SPC can achieve a lower inventory variance ratio than the generalized order-up-to throughout the supply chain except at the retailer. The higher inventory variance at the retailer can be attributed to the higher level of orders smoothing at the retailer $\left(T V A_{\text {ret }}=0.331\right)$ whilst receiving demand of higher variability. This situation can be altered by reducing the width of the sensitivity (safety) zones on the demand and inventory control charts in order to make the IR-SPC policy more sensitive to demand and inventory changes. This is clarified in the sensitivity analysis section.

Table II: Ordering and inventory behaviour in the supply chain under the different policies.

\begin{tabular}{|c|c|c|c|c|c|c|}
\hline \multirow{2}{*}{ Performance measure } & \multicolumn{3}{|c|}{ Smoothing order-up-to } & \multicolumn{3}{c|}{ IR-SPC policy } \\
\cline { 2 - 7 } & $\mathbf{- 9 5} \%$ & Average & $\mathbf{9 5} \%$ & $\mathbf{- 9 5} \%$ & Average & $\mathbf{9 5} \%$ \\
\hline Total variance amplification \\
\hline Retailer & 0.59 & $\mathbf{0 . 6 0 3}$ & 0.61 & 0.31 & $\mathbf{0 . 3 3 1}$ & 0.35 \\
Wholesaler & 0.49 & $\mathbf{0 . 5 0 5}$ & 0.52 & 0.30 & $\mathbf{0 . 3 2 6}$ & 0.35 \\
Distributor & 0.51 & $\mathbf{0 . 5 3 4}$ & 0.56 & 0.35 & $\mathbf{0 . 3 8 2}$ & 0.42 \\
Factory & 0.61 & $\mathbf{0 . 6 5 0}$ & 0.69 & 0.43 & $\mathbf{0 . 4 8 1}$ & 0.53 \\
\hline Inventory variance ratio & \multicolumn{7}{|l|}{} \\
\hline Retailer & 3.53 & $\mathbf{3 . 6 8}$ & 3.83 & 5.08 & $\mathbf{5 . 3 3}$ & 5.57 \\
Wholesaler & 3.90 & $\mathbf{4 . 1 1}$ & 4.33 & 3.78 & $\mathbf{4 . 1 1}$ & 4.45 \\
Distributor & 4.77 & $\mathbf{5 . 0 9}$ & 5.42 & 4.22 & $\mathbf{4 . 6 6}$ & 5.10 \\
Factory & 6.26 & $\mathbf{6 . 7 6}$ & 7.27 & 5.31 & $\mathbf{5 . 9 3}$ & 6.55 \\
\hline
\end{tabular}

\subsection{Comparing IR-SPC with Min-Max inventory policy}

The traditional OUT is a special case of the Min-Max inventory policy in which the minimum and maximum inventory levels are the same. The Min-Max policy is very common in real applications since it is optimal in terms of the inventory costs. Therefore, we conduct a comparison between IR-SPC and Min-Max. The generalized OUT ordering rule presented above can be turned into Min-Max by setting $T n=1$ (see eq. (14)). Similarly, the IR-SPC can also be adapted into Min-Max by setting $K_{I P}^{i}=0$. We consider the following simulation settings to conduct the comparison: $T n=1, m_{i}=30, k_{i}=1, w_{i}=30, n_{i}=15, K_{D}^{i}=2$ and $K_{I P}^{i}=2$. The results of a single simulation run that compares the factory performance under both ordering policies is presented in Fig. 5. The simulation results in Fig. 5 show that IR-SPC is superior compared to Min-Max, in terms of ordering and inventory stability over time.

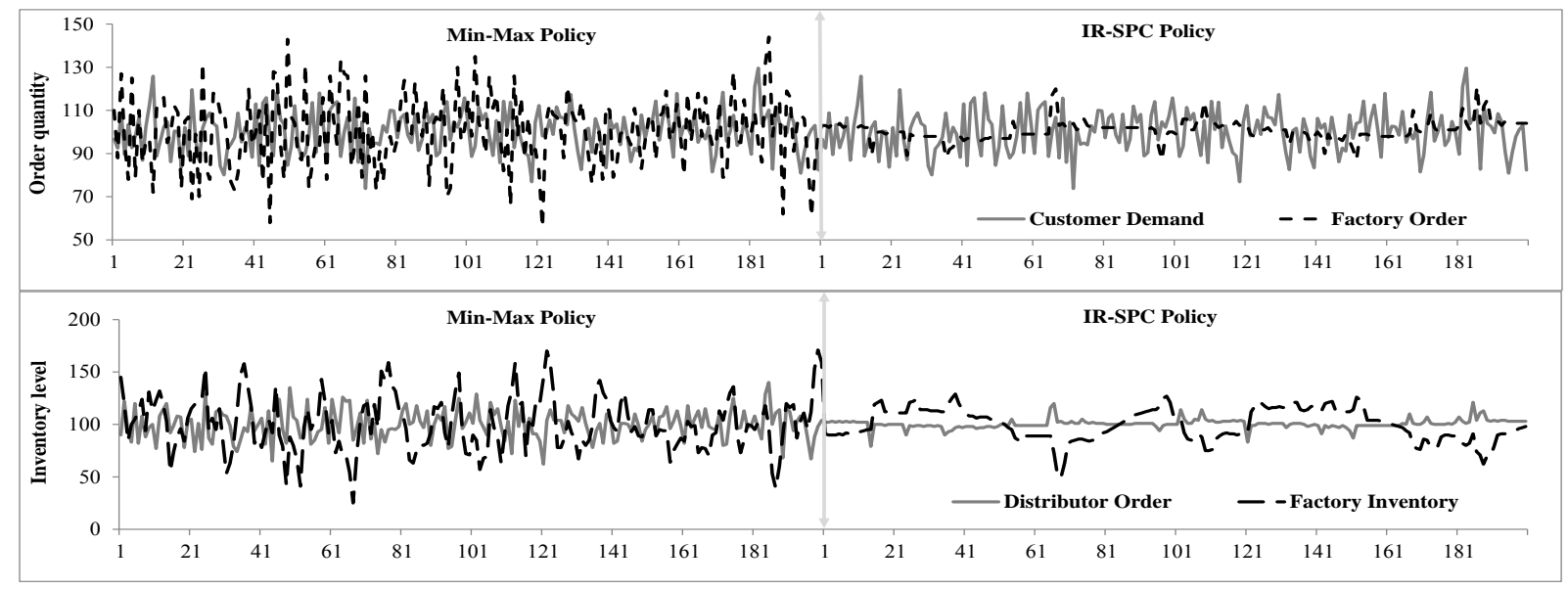

Figure 5: The performance of the factory under both IR-SPC and Min-Max. 
The quantitative comparison results, summarized in Table III, show that the IR-SPC policy outperforms the Min-Max in terms of the bullwhip effect across the supply chain. The bullwhip effect under Min-Max increases considerably from 1.30 at the retailer to 3.36 at the factory while the bullwhip effect under IR-SPC increases from 0.33 at the retailer to 0.48 at the factory. The IR-SPC shows a lower $I n v R_{i}$ compared to the Min-Max except at the retailer. The results show also that the IR-SPC is independent from the initial data employed to run the policy since the supply chain performance under IR-SPC with the smoothing OUT policy is the same as when IR-SPC is applied with the Min-Max.

Table III: A comparison between IR-SPC and Min-Max.

\begin{tabular}{|r|c|c|c|c|}
\hline \multirow{2}{*}{ Echelon } & \multicolumn{2}{|c|}{ Min-Max policy } & \multicolumn{2}{c|}{ IR-SPC policy } \\
\cline { 2 - 5 } & $T V A$ & InvR & TVA & InvR \\
\hline Retailer & $\mathbf{1 . 3 0}$ & 3.50 & $\mathbf{0 . 3 3}$ & 5.33 \\
Wholesaler & $\mathbf{1 . 7 5}$ & 4.68 & $\mathbf{0 . 3 3}$ & 4.11 \\
Distributor & $\mathbf{2 . 4 0}$ & 6.41 & $\mathbf{0 . 3 8}$ & 4.66 \\
Factory & $\mathbf{3 . 3 6}$ & 8.96 & $\mathbf{0 . 4 8}$ & 5.93 \\
\hline
\end{tabular}

\subsection{Sensitivity analysis}

We investigate and compare the sensitivity of both replenishment policies to their operational parameters. In particular, the replenishment policies are evaluated and compared under various combinations of the forecasting and smoothing parameters. The IR-SPC is evaluated under two levels of $K_{D}^{i}$ (forecasting parameter) combined with various levels of $K_{I P}^{i}$ (smoothing parameter), and compared with the OUT policy under two levels of $m_{i}$ combined with two levels of $T n$, i.e., smoothing OUT $(T n=1.618)$ and Min-Max $(T n=1)$. The first comparison is conducted between 'IR-SPC of $K_{D}^{i}=1$ ' and 'OUT policy of $m_{i}=n_{i}=15$ ', thus, both policies are more sensitive to the latest demand changes. The second comparison is the same but with decreasing the sensitivity of the policies to demand changes by setting $m_{i}=w_{i}=30$ for OUT and $K_{D}^{i}=2$ for IR-SPC. The simulation settings are kept the same as in the previous simulation experiments. The comparison results are depicted in Fig. 6.

The results in Fig. 6 show that increasing the moving average parameter $\left(m_{i}\right)$ leads to a decrease in $T V A_{i}$ and $I n v R_{i}$ with improving $A S L_{i}$ at all echelons. The same conclusions are valid for IR-SPC where increasing the level of $K_{D}^{i}$ reduces its sensitivity to demand changes and thus reducing the bullwhip effect. It can be found that the IR-SPC with $K_{I P}^{i}=0$ and $K_{D}^{i}=1$ has a better performance than the OUT with $T n=1$ and $m_{i}=15$ in terms of all performance measures which means that using the demand control chart has been successful to alleviate the impact of the forecasting parameter on the performance measures. The effect of the inventory position control chart becomes prominent as increasing the level of $K_{I P}^{i}$ leads to a decrease in $T V A_{i}$ (at all echelons) and a decrease in $\operatorname{Inv} R_{i}$ at all echelons except at the retailer. The results also show that IR-SPC with $K_{I P}^{i}=0$ and $K_{D}^{i}=2$ achieve the same performance of the OUT with $m_{i}=30$ and $T n=1$. However, when a large value is selected for $m_{i}$, increasing the smoothing level $(T n)$ leads to an increase in $I n v R_{i}$ at the retailer while the inventory stability is improved at the upstream echelons. The same effect happens with IR-SPC with increasing the level of $K_{I P}^{i}$ where the inventory instability increases at the retailer while decreases at the upstream echelons. 

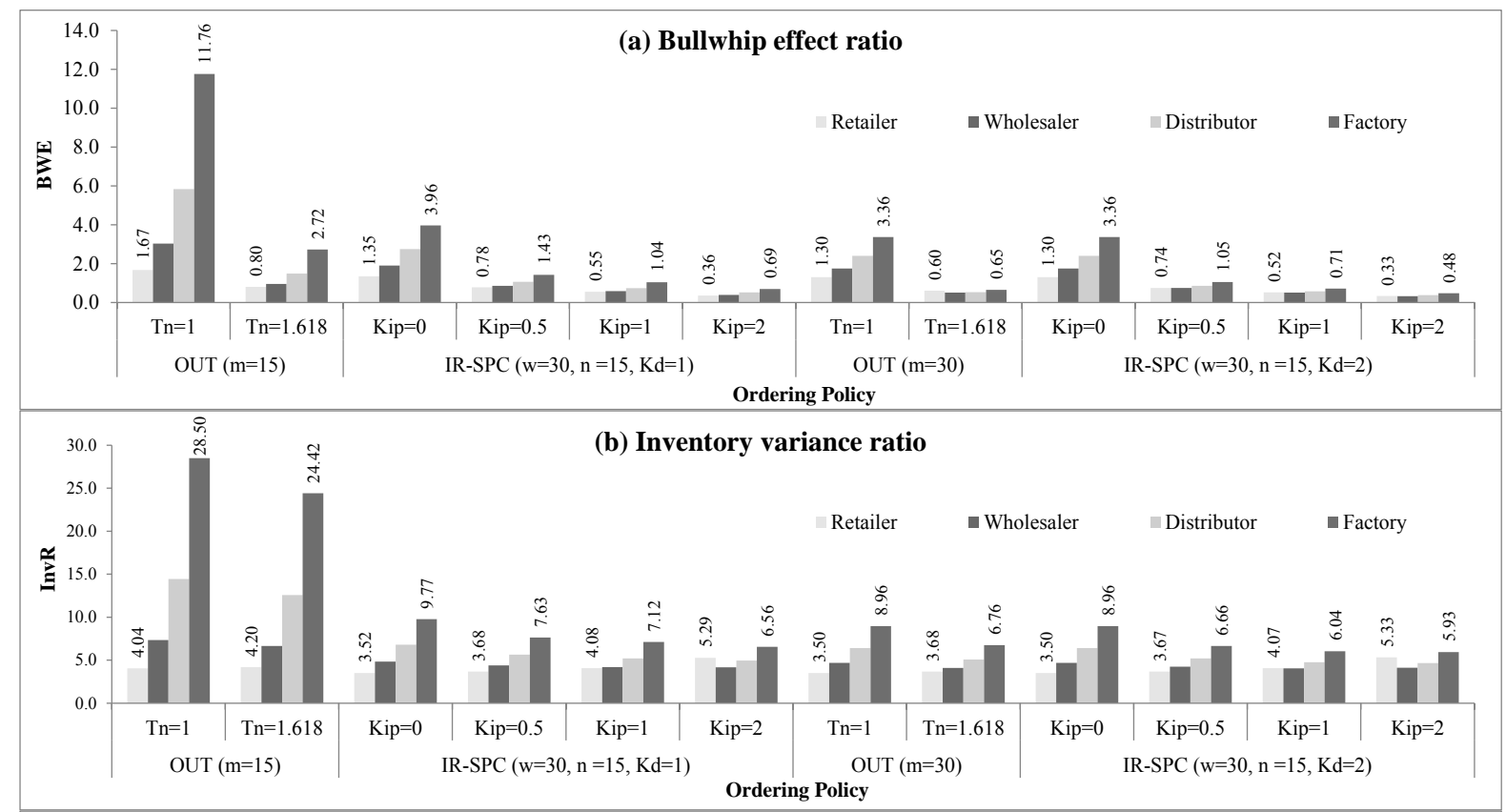

(c) Average service level

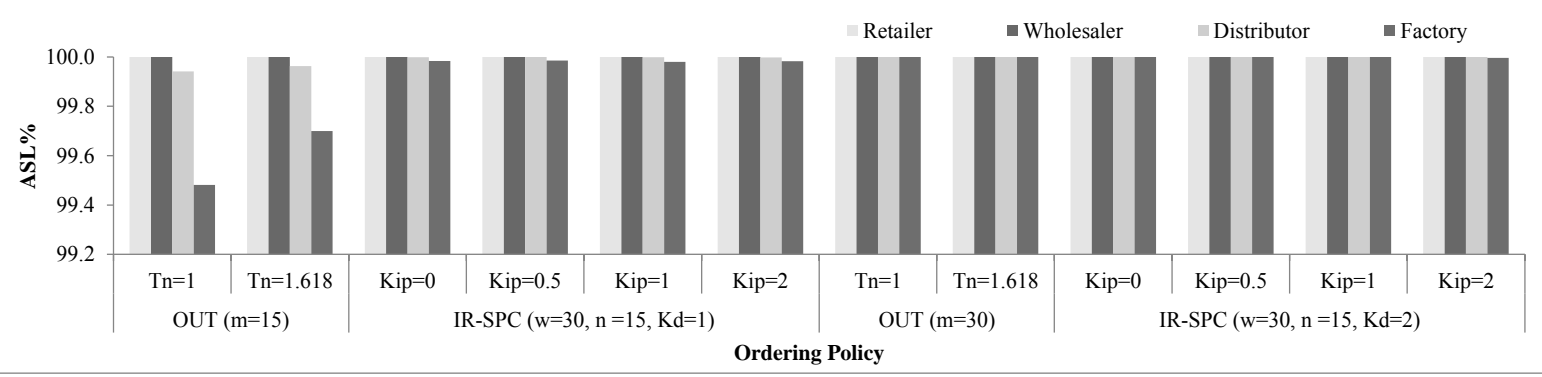

Figure 6: A sensitivity analysis of both IR-SC and OUT to forecasting and smoothing parameters.

\section{DISCUSSION}

This study has introduced a novel inventory control system (IR-SPC) that can be applied (in real-time) to control the ordering and inventory of supply chain echelons in a proper way. The IR-SPC incorporates two control charts to monitor and control the expected demand and inventory position over time. The performance of the IR-SPC has been evaluated and compared with a generalized OUT in a multi-echelon supply chain, through a simulation study.

The simulation results have shown that the IR-SPC has a superior performance compared to the smoothing OUT and Min-Max inventory policies, in the context of a traditional multiechelon supply chain. The results have also shown that the smoothing OUT policy is better than the traditional OUT (Min-Max) where the first policy can achieve a better coordination across the supply chain. The IR-SPC policy has been successful to overcome the effect of short-run fluctuations in comparison to the other inventory control policies. It has also been possible to smooth the orders variability by controlling the parameters of the inventory position control chart. It can be argued that the IR-SPC is a powerful tool to control the bullwhip effect across the supply chain without affecting considerably the inventory performance. Furthermore, the IR-SPC policy has a flexible structure to adapt into the traditional OUT policy.

The IR-SPC has been evaluated in a traditional supply chain and therefore the study can be complemented by evaluating the policy in other supply chain models that allow 
information sharing of customer demand. It is known that supply chain collaboration has a great impact on supply chain operations and currently many supply chains are equipped with technological tools that can allow information visibility and collaboration [14-16, 18, 19, 29].

\section{CONCLUSIONS}

Inventory control policies have been recognized as a contributory factor to supply chain dynamics problems such as bullwhip effect and inventory instability. There is a trade-off between bullwhip effect and inventory performance where decreasing bullwhip effect would increase inventory instability. Therefore, there is a substantial need for inventory control policies that can cope with supply chains dynamics. Recently, control charts have been applied in the area of inventory control and showed a promising performance, however, their effectiveness in multi-echelon supply chains have not been evaluated. In this research, we formulated an inventory control policy (IR-SPC) that relies on control charts to monitor demand changes and control inventory position to be used in dynamic environments like multi-echelon supply chains. We built a simulation model for a four-echelon supply chain in order to evaluate the IR-SPC policy and to compare its performance with an optimized smoothing order-up-to and Min-Max inventory policy. The simulation results showed that the IR-SPC is comparable to and outperforms the other policies in terms of bullwhip effect, inventory variance ratio and average service level. The IR-SPC is generally able to eliminate the bullwhip effect without influencing the inventory performance at any of the supply chain echelons. The promising performance of the IR-SPC would motivate supply chain managers to select it or develop similar replenishment systems for controlling their inventory operations. However, further analysis is still needed to investigate the effectiveness of the IRSPC for other demand patterns and its sensitivity to lead time and other important parameters in supply chains.

\section{REFERENCES}

[1] Lee, H. L.; Padmanabhan, V.; Whang, S. (1997). Information distortion in a supply chain: the bullwhip effect, Management Science, Vol. 43, No. 4, 546-558, doi:10.1287/mnsc.43.4.546

[2] Fisher, M.; Hammond, J.; Obermeyer, W.; Raman, A. (1997). Configuring a supply chain to reduce the cost of demand uncertainty, Production and Operations Management, Vol. 6, No. 3, 211-225, doi:10.1111/j.1937-5956.1997.tb00427.x

[3] Zotteri, G. (2013). An empirical investigation on causes and effects of the bullwhip effect: evidence from the personal care sector, International Journal of Production Economics, Vol. 143, No. 2, 489-498, doi:10.1016/j.ijpe.2012.06.006

[4] Klug, F. (2013). The internal bullwhip effect in car manufacturing, International Journal of Production Research, Vol. 51, No. 1, 303-322, doi:10.1080/00207543.2012.677551

[5] Forrester, J. W. (1958). Industrial dynamics - a major breakthrough for decision makers, Harvard Business Review, Vol. 36, No. 4, 37-66

[6] Sterman, J. D. (1989). Modeling managerial behavior: Misperceptions of feedback in a dynamic decision making experiment, Management Science, Vol. 35, No. 3, 321-339, doi:10.1287/ mnsc.35.3.321

[7] Towill, D. R.; Zhou, L.; Disney, S. M. (2007). Reducing the bullwhip effect: Looking through the appropriate lens, International Journal of Production Economics, Vol. 108, No. 1-2, 444-453, doi:10.1016/j.ijpe.2006.12.024

[8] Burbidge, J. L. (1984). Automated production control with a simulation capability, Proceedings of IFIP Conference, WG 5-7, 1-14

[9] Disney, S. M.; Farasyn, I.; Lambrecht, M.; Towill, D. R.; de Velde, W. V. (2006). Taming the bullwhip effect whilst watching customer service in a single supply chain echelon, European Journal of Operational Research, Vol. 173, No. 1, 151-172, doi:10.1016/j.ejor.2005.01.026 
[10] Buchmeister, B. (2008). Investigation of the bullwhip effect using spreadsheet simulation, International Journal of Simulation Modelling, Vol. 7, No. 1, 29-41, doi:10.2507/IJSIMM07(1) 3.093

[11] Buchmeister, B.; Friscic, D.; Lalic, B.; Palcic, I. (2012). Analysis of a three-stage supply chain with level constraints, International Journal of Simulation Modelling, Vol. 11, No. 4, 196-210, doi:10.2507/IJSIMM11(4)3.212

[12] Costantino, F.; Di Gravio, G.; Shaban, A.; Tronci, M. (2014). SPC-based inventory control policy to improve supply chain dynamics, International Journal of Engineering \& Technology, Vol. 6, No. $1,418-426$

[13] Chen, F.; Drezner, Z.; Ryan, J. K.; Simchi-Levi, D. (2000). Quantifying the bullwhip effect in a simple supply chain: The impact of forecasting, lead times, and information, Management Science, Vol. 46, No. 3, 436-443, doi:10.1287/mnsc.46.3.436.12069

[14] Ciancimino, E.; Cannella, S.; Bruccoleri, M.; Framinan, J. M. (2012). On the bullwhip avoidance phase: the synchronised supply chain, European Journal of Operational Research, Vol. 221, No. 1, 49-63, doi:10.1016/j.ejor.2012.02.039

[15] Costantino, F.; Di Gravio, G.; Shaban, A.; Tronci, M. (2013). Information sharing policies based on tokens to improve supply chain performances, International Journal of Logistics Systems and Management, Vol. 14, No. 2, 133-160, doi:10.1504/IJLSM.2013.051336

[16] Costantino, F.; Di Gravio, G.; Shaban, A.; Tronci, M. (2013). Exploring the bullwhip effect and inventory stability in a seasonal supply chain, International Journal of Engineering Business Management, Vol. 5, 23, 12 pages, doi:10.5772/56833

[17] Dejonckheere, J.; Disney, S. M.; Lambrecht, M. R.; Towill, D. R. (2003). Measuring and avoiding the bullwhip effect: A control theoretic approach, European Journal of Operational Research, Vol. 147, No. 3, 567-590, doi:10.1016/S0377-2217(02)00369-7

[18] Dejonckheere, J.; Disney, S. M.; Lambrecht, M. R.; Towill, D. R. (2004). The impact of information enrichment on the bullwhip effect in supply chains: a control engineering perspective, European Journal of Operational Research, Vol. 153, No. 3, 727-750, doi:10.1016/S0377-2217(02)00808-1

[19] Costantino, F.; Di Gravio, G.; Shaban, A.; Tronci, M. (2014). Replenishment policy based on information sharing to mitigate the severity of supply chain disruption, International Journal of Logistics Systems and Management, Vol. 18, No. 1, 3-23, doi:10.1504/IJLSM.2014.062119

[20] Disney, S. M.; Lambrecht, M. R. (2008). On replenishment rules, forecasting, and the bullwhip effect in supply chains, Foundation and Trends ${ }^{\circledR}$ in Technology, Information and Operations Management, Vol. 2, No. 1, 1-80, doi:10.1561/0200000010

[21] Watts, C. A.; Hahn, C. K.; Sohn, B.-K. (1994). Monitoring the performance of a reorder point system: a control chart approach, International Journal of Operations \& Production Management, Vol. 14, No. 2, 51-61, doi:10.1108/01443579410053211

[22] Pfohl, H. C.; Cullmann, O.; Stolzle, W. (1999). Inventory management with statistical process control: simulation and evaluation, Journal of Business Logistics, Vol. 20, No. 1, 101-120

[23] Cheng, J.-C.; Chou, C.-Y. (2008). A real-time inventory decision system using Western Electric run rules and ARMA control chart, Expert Systems with Applications, Vol. 35, No. 3, 755-761, doi:10.1016/j.eswa.2007.07.019

[24] Lee, H. T.; Wu, J. C. (2006). A study on inventory replenishment policies in a two-echelon supply chain system, Computers \& Industrial Engineering, Vol. 51, No. 2, 257-263, doi:10.1016/j.cie.2006.01.005

[25] Montgomery, D. C. (1996). Introduction to Statistical Quality Control, Wiley, New York

[26] Chatfield, D. C.; Kim, J. G.; Harrison, T. P.; Hayya, J. C. (2004). The bullwhip effect - impact of stochastic lead time, information quality, and information sharing: a simulation study, Production and Operations Management, Vol. 13, No. 4, 340-353, doi:10.1111/j.1937-5956.2004.tb00222.x

[27] Disney, S. M.; Towill, D. R. (2003). On the bullwhip and inventory variance produced by an ordering policy, Omega, Vol. 31, No. 3, 157-167, doi:10.1016/S0305-0483(03)00028-8

[28] Zipkin, P. H. (2000). Foundations of Inventory Management, McGraw-Hill, New York

[29] Sobottka, T.; Leitner, R.; Sihn, W. (2012). Methodology for the development of RFID value added services to improve supply chain operations, Transactions of FAMENA, Vol. 36, No. 2, $67-77$ 